Document downloaded from:

http://hdl.handle.net/10251/105894

This paper must be cited as:

Hernández-Verón, MA.; Martínez Molada, E.; Teruel-Ferragud, C. (2017). Semilocal convergence of a k-step iterative process and its application for solving a special kind of conservative problems. Numerical Algorithms. 76(2):309-331. doi:10.1007/s11075-0160255-z

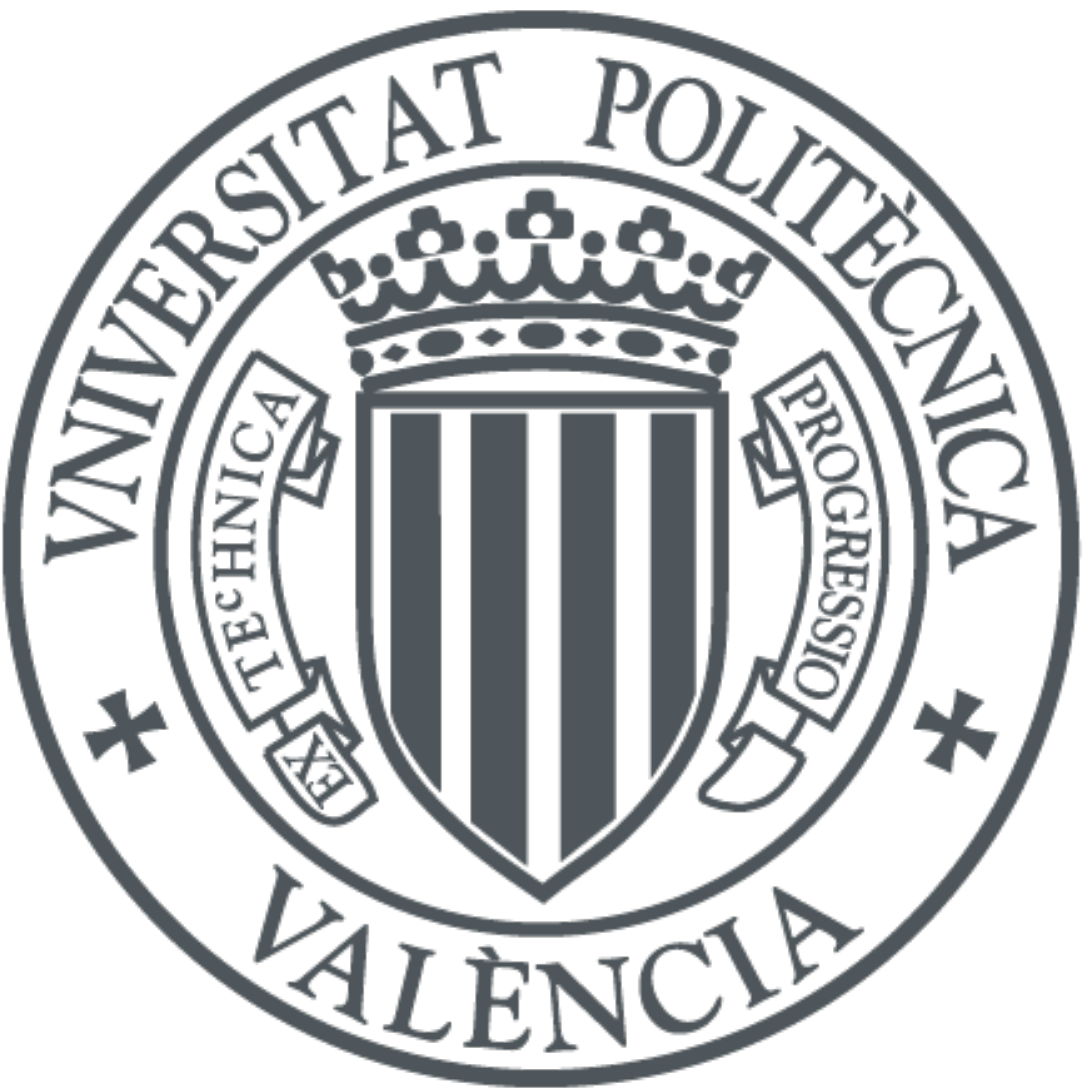

The final publication is available at

http://doi.org/10.1007/s11075-016-0255-z

Copyright Springer-Verlag

Additional Information 


\title{
Semilocal convergence of a k-step iterative process and its application for solving a special kind of conservative problems
}

\author{
M. A. Hernández-Verón ${ }^{\mathrm{a}}$, Eulalia Martínez ${ }^{\mathrm{b}}$, Carles Teruel $^{\mathrm{b}}$. \\ ${ }^{\text {a }}$ Dpt. of Mathematics and Computation, University of La Rioja. \\ 26004 Logroño, Spain. \\ E-mail address: mahernan@unirioja.es

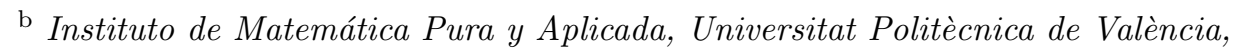 \\ 46022 Valencia, Spain. \\ E-mail address: eumarti@mat.upv.es
}

\begin{abstract}
In this paper, we analyze the semilocal convergence of k-steps Newton's method with frozen first derivative in Banach spaces. The method reaches order of convergence $k+1$. By imposing only the assumption that the Fréchet derivative satisfies the Lipschitz continuity we define appropriate recurrence relations for obtaining the domains of convergence and uniqueness.

We also define the accessibility regions for this iterative process in order to guarantee the semilocal convergence and perform a complete study of their efficiency. Our final aim is to apply these theoretical results to solve a special kind of conservative systems.
\end{abstract}

Keywords: Nonlinear equations, order of convergence, iterative methods, semilocal convergence, Conservative systems.

2000 Mathematics Subject Classification: 47H99, 65H10.

Preparation of this paper was partly supported by the Ministry of Science and Innovation (MTM2014-52016-C2-01-02). 


\section{Introduction}

Many physical, biological, social, etc. phenomena can be modeled by initial value problems, partial derivative equations and integral equations, like, among many others, planetary motion, Newtonian mechanics, chemical reacts problems, electric circuits, heat conduction, vibratory motion, transmission of signals, problems on population growth, studies about gender-based violence, different types of rural crimes, etc. See, for instance, [2] and [16]. These differential equations, in most cases can not be solved analytically and then by using a adequate numerical method the problem is transformed in nonlinear equations.

We center our attention on conservative system like gravitational and electric fields. System in which work done by a force is independent of the path taken and is equal to the difference between the final and initial values of an energy function.

Specifically we consider conservative systems where the damping force is null and then can be described as follows:

$$
m \frac{d^{2} x(t)}{d t^{2}}+\phi(x(t))=0
$$

where $\mathrm{m}$ is the mass on which acts a nonlinear force $\phi(x(t))$ in an interval $[a, b]$ verifying $x(a)=A, x(b)=B$.

Our aim is to use techniques of functional analysis in order to obtain domains that contains the solution of such problems, these domains are obtained in the infinite dimensional space that the function $x(t)$ belongs to. Uniqueness conditions for these domains are also established. These are done by using iterative methods for nonlinear equations defined in Banach spaces.

This paper focuses on the resolution of these nonlinear equations $F(x)=$ 0 . The solutions of this equation, again, can rarely be found in a closed form, so that we usually look for numerical approximations. As a consequence, the methods for solving the previous equation are usually iterative. So, starting from one initial approximation of a solution $x^{*}$ of the equation $F(x)=0$, a sequence $\left\{x_{n}\right\}$ of approximations is constructed such that the sequence $\left\{\left\|x_{n}-x^{*}\right\|\right\}$ is decreasing and a better approximation to the solution $x^{*}$ is then obtained at every step. Obviously, the interest focuses on $\lim _{n} x_{n}=x^{*}$.

To give sufficient generality to the problem of approximating a solution of a nonlinear equation, we consider equations of the form $F(x)=0$, where $F$ is a nonlinear operator, $F: \Omega \subseteq X \longrightarrow Y$, defined on a nonempty open convex domain $\Omega$ of a Banach space $X$ with values in a Banach space $Y$, for this purpose see [10] and [13]. 
In relation to the above, we can obtain the sequence of approximations $\left\{x_{n}\right\}$ by different ways, depending on the iterative methods that are applied. Between these, the best-known iteration is Newton's method, whose algorithm is the following:

$$
x_{0} \text { given in } \Omega, \quad x_{n+1}=x_{n}-\left[F^{\prime}\left(x_{n}\right)\right]^{-1} F\left(x_{n}\right), \quad n=0,1,2 \ldots
$$

The choice of a method for solving $F(x)=0$ usually depends on its efficiency, which links the speed of convergence (order of convergence) of the method to its computational cost. Two classic measurements of the efficiency, in the sense defined by Traub [17] and Ostrowski [14], are the efficiency index $(E I)$ and the computatioal efficiency $(C E)$, which are respectively defined by

$$
E I=\rho^{1 / a} \quad \text { and } \quad C E=\rho^{1 / p},
$$

where $\rho$ is the order of convergence of the method, $a$ represents the number of the evaluations of functions necessary to apply the method and $p$ is the number of operations (products and divisions) that are needed to compute each iteration of the method.

If we consider one-point iterative methods without memory, i.e., $x_{n+1}=$ $G\left(x_{n}\right)$ with $x_{0}$ given in $\Omega$, as Newton's method (1), it is known that their order of convergence $\rho$ is a natural number and, moreover, the algorithm of these methods depend explicitly of the first $\rho-1$ derivatives of the function involved in the equation. So, if we want consider iterative processes with high order of convergence, the computational cost increases as it is necessary to evaluate the successive derivatives of the function involved in the algorithm of the method. Then, in this paper, we are interested in numerical methods that avoid the expensive computation of the derivatives of the function $F$ at each step but high order of convergence is reached. Therefore, we consider a k-steps iterative process with frozen first derivative given by the following algorithm:

$$
\left\{\begin{array}{l}
x_{0} \in D, \\
x_{n}^{(1)}=x_{n}^{(0)}-\Gamma_{n} F\left(x_{n}^{(0)}\right), \\
x_{n}^{(2)}=x_{n}^{(1)}-\Gamma_{n} F\left(x_{n}^{(1)}\right), \\
\quad \vdots \\
x_{n}^{(k-1)}=x_{n}^{(k-2)}-\Gamma_{n} F\left(x_{n}^{(k-2)}\right), \\
x_{n}^{(k)}=x_{n}^{(k-1)}-\Gamma_{n} F\left(x_{n}^{(k-1)}\right), \quad n \geqslant 0,
\end{array}\right.
$$

where $\Gamma_{n}=F^{\prime}\left(x_{n}\right)^{-1}, x_{n}=x_{n}^{(0)}$ and $x_{n+1}=x_{n}^{(k)}$, with $k \geq 1$. It is well known that if we compose Newton's method with itself $k$ times but with frozen the derivative, we obtain a method of order $k+1$, this is a classical 
result obtained by Traub, [17]. Moreover, being a iterative process of high order, does not increase the expensive computation of derivatives because this iterative process only uses a first derivative in each step.

Three types of studies can be done when we are interested in proving the convergence of sequence (3): local, semilocal and global. First, the local study of the convergence is based on demanding conditions to the solution $x^{*}$, from certain conditions on the operator $F$, and provide the so-called ball of convergence of (3), that shows the accessibility to $x^{*}$ from the initial approximation $x_{0}$ belonging to the ball, see [3] and [4]. Second, the semilocal study of the convergence is based on demanding conditions to the initial approximation $x_{0}$, from certain conditions on the operator $F$, and provide the so-called domain of parameters corresponding to the conditions required to the initial approximation that guarantee the convergence of the sequence generates by the iterative method to the solution $x^{*}$, see [5] and [7]. Third, the global study of the convergence guarantees, from certain conditions on the operator $F$, the convergence of the sequence to the solution $x^{*}$ in a domain and independently of the initial approximation $x_{0}$, see [15] and [8]. The three studies demand conditions on the operator $F$. However, requirement of conditions to the solution, to the initial approximation, or to none of these, determines the different types of studies.

The local study of the convergence has the disadvantage of being able to guarantee that the solution, that is unknown, can satisfy certain conditions. In general, the global study of the convergence is very specific as regards the type of operators to consider, as a consequence of absence of conditions on the initial approximations and on the solution.

We emphasize now that, although some authors have published k-steps iterative methods, like in [6] and [1], as far as we know the semilocal convergence study has not been performed for these general methods. This is the aim of our studies nowadays.

In this work, we focus our attention on the analysis of the semilocal convergence of k-steps Newton's method with frozen first derivative (3). For this purpose only conditions on $F^{\prime}$ are imposed, however we can reach order of convergence $k+1$. Our final aim is to apply these theoretical results to solve efficiently a special kind of conservative systems.

The rest of paper is organized as follows. In section 2 we present previous results and define appropriate recurrence relations for establishing the semilocal convergence study for this k-steps method that is completed in section 3. Then, section 4 is devoted to define the accessibility regions for this iterative process. Finally in section 5 we apply the theoretical results obtained in order to solve a special case of conservative systems with maximum efficiency. 


\section{Semilocal convergence}

The most popular semilocal convergence result for Newton's method (1) is the variant of the Newton-Kantorovich theorem [10] given by Ortega in [13], which is established under the following conditions:

$\left(C_{1}\right)$ There exists $\Gamma_{0}=\left[F^{\prime}\left(x_{0}\right)\right]^{-1} \in \mathcal{L}(Y, X)$, for some $x_{0} \in \Omega$, with $\left\|\Gamma_{0}\right\| \leq \beta$ and $\left\|\Gamma_{0} F\left(x_{0}\right)\right\| \leq \eta$, where $\mathcal{L}(Y, X)$ is the set of bounded linear operators from $Y$ to $X$.

$\left(C_{2}\right)$ There exists a constant $K \geq 0$ such that $\left\|F^{\prime}(x)-F^{\prime}(y)\right\| \leq$ $K\|x-y\|$ for $x, y \in \Omega$.

$\left(C_{3}\right) h=K \beta \eta \leq \frac{1}{2}$.

Together with $B\left(x_{0}, r \eta\right) \subset \Omega$, where $r=\frac{1-\sqrt{1-2 h}}{h}$ is the smallest positive zero of the polynomial $p(t)=\frac{K}{2} t^{2}-\frac{t}{\beta}+\frac{\eta}{\beta}$, guarantees the semilocal convergence of Newton's method.

Now, our aim it is to establish the semilocal convergence study for this ksteps method (3), demanding previous conditions to the initial approximation $x_{0}$ and from the operator $F$, i.e., let us assume the conditions $\left(C_{1}\right)$ and $\left(C_{2}\right)$. So, fixed a number of steps $k \in \mathbb{N}$, we obtain a semilocal result for a iterative process (3) of order $k+1, k \geq 1$, in the same initial conditions that for an iterative process of second order, the Newton's method (1). For this, we modify condition $\left(C_{3}\right)$ of Ortega. Observe that condition $\left(C_{1}\right)$, required to the initial approximation $x_{0}$, define the parameters $\beta_{0}$ and $\eta_{0}$, and condition $\left(C_{2}\right)$, required to the operator $F$, define the fixed parameter $K$. So, from these parameters, we modify the condition $\left(C_{3}\right)$.

We state the semilocal convergence result for the k-steps iterative method proposed in the following terms.

Theorem 1 Let $F$ is a nonlinear operator, $F: \Omega \subseteq X \longrightarrow Y$, defined on a nonempty open convex domain $\Omega$ of a Banach space $X$ with values in a Banach space $Y$. Suppose that conditions $\left(C_{1}\right)-\left(C_{2}\right)$ are satisfied. Let

$$
S_{0}(t)=\left\{\begin{array}{l}
1 \quad \text { if } \mathrm{k}=1 \\
1+\frac{1}{2} h_{0}+\frac{1}{2} h_{0}^{2} t+\ldots+\frac{1}{2} h_{0}^{k-1} t^{k-2} \text { if } \mathrm{k}>1
\end{array}\right.
$$

Then, For a fixed number of steps $k$, if the equation

$$
t=S_{0}(t)\left[1+\frac{h_{0}^{k} t^{k-1}}{\left(1-h_{0} t\right)\left(2-h_{0}^{k} t^{k-1}\right)}\right]
$$


has at least one positive real root and the smallest positive real root, denoted by $R$, satisfies

$$
\frac{h_{0}}{2\left(1-h_{0} R\right)^{2}}<1
$$

and $B\left(x_{0}, R \eta\right) \subset \Omega$, then iterative process given by (3), converges to a solution $x^{*}$ of the equation $F(x)=0$, starting at $x_{0}$, and $x_{n}^{(j)}, x^{*} \in \overline{B\left(x_{0}, R \eta\right)}$, for $1 \leq j \leq k$ and for all $n \in \mathbb{N}$. Moreover the solution $x^{*}$ is unique in $B\left(x_{0}, \frac{2}{K \beta}-R \eta\right) \cap \Omega$.

Before proving the main result we need to obtain the following lemmas based on the recurrence relations for the state conditions.

\section{$2.1 \quad$ Recurrence relations}

We will first analyze the well definition of iterative process (3) for different numbers of steps, $1 \leq j \leq k, k \geq 1$. For these, we obtain the following result.

Lemma 2 Let us assume that exists $R>1$ such that $x_{n}^{(j)} \in B\left(x_{0}, R \eta\right)$, with $B\left(x_{0}, R \eta\right) \subset \Omega$, for $1 \leq j \leq k, k \geq 1$ and $n \in \mathbb{N}$. Then, for $R<\frac{1}{\beta K \eta}$, the iterative process (3) is well defined.

\section{Proof:}

In first place, obviously, $F\left(x_{n}^{(j)}\right)$ is well defined for all $n \in \mathbb{N}$ and $1 \leq j \leq k$, $k \geq 1$.

On the other hand, it is necessary that $\Gamma_{n}=\left[F^{\prime}\left(x_{n}\right)\right]^{-1}$ exists for all $n \in \mathbb{N}$. For that purpose we obtain:

$$
\left\|I-\Gamma_{0} F^{\prime}\left(x_{n}\right)\right\| \leq\left\|\Gamma_{0}\right\|\left\|F^{\prime}\left(x_{n}\right)-F^{\prime}\left(x_{0}\right)\right\| \leq \beta K\left\|x_{n}-x_{0}\right\|<\beta K \eta R .
$$

Then, if $\beta K \eta R<1$ we apply Banach's lemma and deduce the existence of $\Gamma_{n}$ and it verifies:

$$
\left\|\Gamma_{n}\right\| \leq \frac{\beta}{1-\beta K \eta R}
$$

Now, we are interested to obtain the recurrence relations necessary to prove the semilocal convergence of iterative process (3). From now, let us assume the hypotheses of previous lemma.

In these conditions, for $n=0$ and $j=1$, we have:

$$
\left\|x_{0}^{(1)}-x_{0}^{(0)}\right\| \leq\left\|\Gamma_{0} F\left(x_{0}^{(0)}\right)\right\| \leq \eta .
$$


We will use the following notation:

$$
\begin{aligned}
\eta_{0} & =\eta, \\
\beta_{0} & =\beta, \\
h_{0} & =\beta_{0} K \eta_{0} .
\end{aligned}
$$

Now, by considering the Taylor expansion of $F\left(x_{0}^{(1)}\right)$ around $x_{0}^{(0)}$ and using first step of (3), we have:

$F\left(x_{0}^{(1)}\right)=\int_{x_{0}^{(0)}}^{x_{0}^{(1)}}\left[F^{\prime}(z)-F^{\prime}\left(x_{0}^{(0)}\right)\right] \mathrm{d} z=\int_{0}^{1}\left[F^{\prime}\left(x_{0}+\tau\left(x_{0}^{(1)}-x_{0}^{(0)}\right)\right)-F^{\prime}\left(x_{0}\right)\right] \mathrm{d} \tau\left(x_{0}^{(1)}-x_{0}^{(0)}\right)$,

then, by taking norms and using $\left(C_{2}\right)$ we get:

$$
\left\|F\left(x_{0}^{(1)}\right)\right\| \leq \frac{1}{2} K \eta_{0}\left\|x_{0}^{(1)}-x_{0}^{(0)}\right\|,
$$

so, it follows:

$$
\left\|x_{0}^{(2)}-x_{0}^{(1)}\right\|=\left\|\Gamma_{0}\right\|\left\|F\left(x_{0}^{(1)}\right)\right\| \leq \frac{1}{2} h_{0}\left\|x_{0}^{(1)}-x_{0}^{(0)}\right\|,
$$

and

$$
\left\|x_{0}^{(2)}-x_{0}^{(0)}\right\| \leq\left\|x_{0}^{(2)}-x_{0}^{(1)}\right\|+\left\|x_{0}^{(1)}-x_{0}^{(0)}\right\| \leq\left(1+\frac{1}{2} h_{0}\right) \eta_{0}
$$

In the same way, for $j=2$, we bound $F\left(x_{0}^{(2)}\right)$ by a similar process. First, we obtain:

$F\left(x_{0}^{(2)}\right)=\int_{x_{0}^{(1)}}^{x_{0}^{(2)}}\left[F^{\prime}(z)-F^{\prime}\left(x_{0}\right)\right] \mathrm{d} z=\int_{0}^{1}\left[F^{\prime}\left(x_{0}^{(1)}+\tau\left(x_{0}^{(2)}-x_{0}^{(1)}\right)\right)-F^{\prime}\left(x_{0}^{(0)}\right)\right] \mathrm{d} \tau\left(x_{0}^{(2)}-x_{0}^{(1)}\right)$,

and, by taking norms and using $\left(C_{2}\right)$, we get:

$$
\left\|F\left(x_{0}^{(2)}\right)\right\| \leq K R \eta_{0}\left\|x_{0}^{(2)}-x_{0}^{(1)}\right\|,
$$

where we have used that $x_{0}^{(1)}+\tau\left(x_{0}^{(2)}-x_{0}^{(1)}\right) \in B\left(x_{0}, R \eta\right)$, since $x_{0}^{(1)}, x_{0}^{(2)} \in$ $B\left(x_{0}, R \eta\right)$ and its convexity's property. By using this bound we have:

$$
\left\|x_{0}^{(3)}-x_{0}^{(2)}\right\| \leq\left\|\Gamma_{0}\right\|\left\|F\left(x_{0}^{(2)}\right)\right\| \leq h_{0}\left\|x_{0}^{(2)}-x_{0}^{(1)}\right\| \leq \frac{1}{2} h_{0}^{2} R\left\|x_{0}^{(1)}-x_{0}^{(0)}\right\|,
$$


and

$$
\left\|x_{0}^{(3)}-x_{0}\right\| \leq\left\|x_{0}^{(3)}-x_{0}^{(2)}\right\|+\left\|x_{0}^{(2)}-x_{0}^{(0)}\right\| \leq\left(1+\frac{1}{2} h_{0}+\frac{1}{2} h_{0}^{2} R\right) \eta_{0},
$$

Then, from the previous reasonings, we can obtain the following result.

Lemma 3 For $n=0$, the following relations are verified for $2 \leq j \leq k-1$ :

i) $\left\|F\left(x_{0}^{(j)}\right)\right\| \leq K R \eta_{0}\left\|x_{0}^{(j)}-x_{0}^{(j-1)}\right\|$,

ii) $\left\|x_{0}^{(j)}-x_{0}^{(j-1)}\right\| \leq h_{0} R\left\|x_{0}^{(j-1)}-x_{0}^{(j-2)}\right\|$,

iii) $\left\|x_{0}^{(j)}-x_{0}^{(0)}\right\| \leq\left(1+\frac{1}{2} h_{0}+\frac{1}{2} h_{0}^{2} R+\ldots+\frac{1}{2} h_{0}^{j-1} R^{j-2}\right) \eta_{0}$.

Then, these relations are verified for $j=k$.

Proof: By considering the Taylor expansion of $F\left(x_{0}^{(j)}\right)$ around $x_{0}^{(j-1)}$ and using $j-$ th step of (3), we have:

$F\left(x_{0}^{(j)}\right)=\int_{x_{0}^{(j-1)}}^{x_{0}^{(j)}}\left[F^{\prime}(z)-F^{\prime}\left(x_{0}\right)\right] \mathrm{d} z=\int_{0}^{1}\left[F^{\prime}\left(x_{0}^{(j-1)}+\tau\left(x_{0}^{(j)}-x_{0}^{(j-1)}\right)\right)-F^{\prime}\left(x_{0}\right)\right] \mathrm{d} \tau\left(x_{0}^{(j)}-x_{0}^{(j-1)}\right)$,

then, by taking norms and using that $x_{0}^{(j-1)}+\tau\left(x_{0}^{(j)}-x_{0}^{(j-1)}\right) \in B\left(x_{0}, R \eta\right)$, since $x_{0}^{(j)}, x_{0}^{(j-1)} \in B\left(x_{0}, R \eta\right)$, one obtains:

$$
\left\|F\left(x_{0}^{(j)}\right)\right\| \leq K R \eta_{0}\left\|x_{0}^{(j)}-x_{0}^{(j-1)}\right\|,
$$

So that,

$$
\begin{aligned}
\left\|x_{0}^{(j+1)}-x_{0}^{(j)}\right\| & \leq\left\|\Gamma_{0}\right\|\left\|F\left(x_{0}^{(j)}\right)\right\| \leq h_{0}\left\|x_{0}^{(j)}-x_{0}^{(j-1)}\right\| \leq h_{0}^{2} R^{2}\left\|x_{0}^{(j-1)}-x_{0}^{(j-2)}\right\| \\
& \leq \ldots \leq h_{0}^{j-1} R^{j-1}\left\|x_{0}^{(2)}-x_{0}^{(1)}\right\| \leq \frac{1}{2} h_{0}^{j} R^{j-1}\left\|x_{0}^{(1)}-x_{0}^{(0)}\right\|,
\end{aligned}
$$

and

$\left\|x_{0}^{(j+1)}-x_{0}^{(0)}\right\| \leq\left\|x_{0}^{(j+1)}-x_{0}^{(j)}\right\|+\left\|x_{0}^{(j)}-x_{0}^{(0)}\right\| \leq\left(1+\frac{1}{2} h_{0}+\frac{1}{2} h_{0}^{2} R+\ldots+\frac{1}{2} h_{0}^{j} R^{j-1}\right) \eta_{0}$,

Then, the result is proved.

Moreover, for $j=k$, we can be obtain:

$$
\begin{aligned}
\left\|F\left(x_{0}^{(k)}\right)\right\| & \leq K R \eta\left\|x_{0}^{(k)}-x_{0}^{(k-1)}\right\|, \\
\left\|x_{0}^{(k)}-x_{0}^{(k-1)}\right\| & \leq h_{0} R\left\|x_{0}^{(k-1)}-x_{0}^{(k-2)}\right\|, \\
\left\|x_{0}^{(k)}-x_{0}^{(0)}\right\| & =\left\|x_{1}-x_{0}\right\| \leq\left(1+\frac{1}{2} h_{0}+\frac{1}{2} h_{0}^{2} R+\ldots+\frac{1}{2} h_{0}^{k-1} R^{k-2}\right) \eta_{0},
\end{aligned}
$$


Therefore, if we consider

$$
S_{0}(R)=\left\{\begin{array}{l}
1 \quad \text { if } \mathrm{k}=1 \\
1+\frac{1}{2} h_{0}+\frac{1}{2} h_{0}^{2} R+\ldots+\frac{1}{2} h_{0}^{k-1} R^{k-2} \text { if } \mathrm{k}>1
\end{array}\right.
$$

from previous results, we have that $x_{0}^{(j)}, 1 \leq j \leq k-1$ and $x_{1}=x_{0}^{(k)}$ belong to $B\left(x_{0}, S_{0}(R) \eta_{0}\right)$.

Now, we analyze the method (3) for $n=1$. First of all, from lemma 2 we obtain the existence of $\Gamma_{1}=\left[F^{\prime}\left(x_{1}\right)\right]^{-1}$ and denote

$$
\left\|\Gamma_{1}\right\| \leq \frac{\beta}{1-h_{0} R} \equiv \beta_{1} .
$$

So, we can define $x_{1}^{(1)}=x_{1}^{(0)}-\Gamma_{1} F\left(x_{1}^{(0)}\right)$, with $x_{1}^{(0)}=x_{0}^{(k)}=x_{1}$, and we have:

$$
\begin{aligned}
\left\|x_{1}^{(1)}-x_{1}^{(0)}\right\| & \leq\left\|\Gamma_{1} F\left(x_{1}^{(0)}\right)\right\| \leq \beta_{1} K R \eta_{0}\left\|x_{0}^{(k)}-x_{0}^{(k-1)}\right\| \\
& \leq \beta_{1} K R \eta_{0} \frac{1}{2}\left(\beta K \eta_{0}\right)^{k-1} R^{k-2}\left\|x_{0}^{(1)}-x_{0}\right\| \\
& \leq \frac{\beta_{0} K R \eta_{0}}{2\left(1-h_{0} R\right)} h_{0}^{k-1} R^{k-2} \eta_{0}=\frac{1}{2\left(1-h_{0} R\right)} h_{0}^{k} R^{k-1} \eta_{0} .
\end{aligned}
$$

Then, by taking $\eta_{1}=\frac{1}{2\left(1-h_{0} R\right)} h_{0}^{k} R^{k-1} \eta_{0}$ we have the same conditions that in before step, that is:

$$
\left\{\begin{array}{l}
\left\|\Gamma_{1}\right\| \leq \beta_{1} \\
\left\|\Gamma_{1} F\left(x_{1}\right)\right\| \leq \eta_{1},
\end{array}\right.
$$

Then, following with the same previous notation, we consider $h_{1}=\beta_{1} K \eta_{1}$ and

$$
S_{1}(R)=\left\{\begin{array}{l}
1 \quad \text { if } \mathrm{k}=1 \\
1+\frac{1}{2} h_{1}+\frac{1}{2} h_{1}^{2} R+\ldots+\frac{1}{2} h_{1}^{k-1} R^{k-2} \text { if } \mathrm{k}>1
\end{array}\right.
$$

so, by same previous reasoning, we establish:

$$
\begin{aligned}
& \left\|F\left(x_{1}^{(1)}\right)\right\| \leq \frac{1}{2} K \eta_{1}\left\|x_{1}^{(1)}-x_{1}^{(0)}\right\|, \\
& \left\|F\left(x_{1}^{(j)}\right)\right\| \leq K R \eta_{1}\left\|x_{1}^{(j)}-x_{1}^{(j-1)}\right\|, \quad 2 \leq j \leq k,
\end{aligned}
$$

and moreover, for $1 \leq j \leq k-1$, it holds:

$$
\begin{aligned}
& \left\|x_{1}^{(j+1)}-x_{1}^{(j)}\right\| \leq h_{1} R\left\|x_{1}^{(j)}-x_{1}^{(j-1)}\right\| \leq \frac{1}{2} h_{1}^{j} R^{j-1}\left\|x_{1}^{(j)}-x_{1}^{(j-1)}\right\|, \\
& \left\|x_{1}^{(j+1)}-x_{1}^{(0)}\right\| \leq\left(1+\frac{1}{2} h_{1}+\frac{1}{2} h_{1}^{2} R+\ldots+\frac{1}{2} h_{1}^{j-1} R^{j-2}\right) \eta_{1} .
\end{aligned}
$$


Then, it follows:

$$
\left\|F\left(x_{1}^{(k)}\right)\right\| \leq K R \eta_{1}\left\|x_{1}^{(k)}-x_{1}^{(k-1)}\right\|,
$$

and

$$
\begin{aligned}
& \left\|x_{1}^{(k)}-x_{1}^{(k-1)}\right\|=\left\|x_{2}-x_{1}^{(k-1)}\right\| \leq h_{1} R\left\|x_{1}^{(k-1)}-x_{1}^{(k-2)}\right\| \leq \frac{1}{2} h_{1}^{k-1} R^{k-2}\left\|x_{1}^{(1)}-x_{1}^{(0)}\right\|, \\
& \left\|x_{1}^{(k)}-x_{1}^{(0)}\right\|=\left\|x_{2}-x_{1}\right\| \leq S_{1}(R) \eta_{1} .
\end{aligned}
$$

Then, $x_{1}^{(j)}, x_{2} \in B\left(x_{1}, S_{1}(R) \eta_{1}\right)$, for $1 \leq j \leq k-1$, being $x_{2}=x_{1}^{(k)}$.

Now we can go on with $n=2$. First of all, from lemma 2, we obtain the existence of $\Gamma_{2}=\left[F^{\prime}\left(x_{2}\right)\right]^{-1}$ and denote

$$
\left\|\Gamma_{2}\right\| \leq \frac{\beta}{1-h_{0} R} \equiv \beta_{2} .
$$

Let us observe that $\beta_{2}=\beta_{1}$.

For $j=1$, having into account that $x_{2}=x_{2}^{(0)}$, we have:

$$
\begin{aligned}
\left\|x_{2}^{(1)}-x_{2}^{(0)}\right\| & \leq\left\|\Gamma_{2} F\left(x_{2}^{(0)}\right)\right\| \leq \beta_{2} K R \eta_{1}\left\|x_{1}^{(k)}-x_{1}^{(k-1)}\right\| \leq \\
& \beta_{2} K R \eta_{1} \frac{1}{2}\left(\beta_{1} K \eta_{1}\right)^{k-1} R^{k-2}\left\|x_{1}^{(1)}-x_{1}^{(0)}\right\| \leq \frac{1}{2} h_{1}^{k} R^{k-1} \eta_{1} .
\end{aligned}
$$

Then, by taking $\eta_{2}=\frac{1}{2} h_{1}^{k} R^{k-1} \eta_{1}$, we have a similar situation that in the previous case $n=1$, see $(7)$ :

$$
\left\{\begin{array}{l}
\left\|\Gamma_{2}\right\| \leq \beta_{2} \\
\left\|\Gamma_{2} F\left(x_{2}\right)\right\| \leq \eta_{2}
\end{array}\right.
$$

then, by a similar development than the performed previously, assuming the hypotheses of lemma 2 , and following with the same previous notation, we consider $h_{2}=\beta_{2} K \eta_{2}$ and

$$
S_{2}(R)=\left\{\begin{array}{l}
1 \text { if } \mathrm{k}=1 \\
1+\frac{1}{2} h_{2}+\frac{1}{2} h_{2}^{2} R+\ldots+\frac{1}{2} h_{2}^{k-1} R^{k-2} \text { if } \mathrm{k}>1
\end{array}\right.
$$

we establish that $x_{2}^{(j)}, x_{3} \in B\left(x_{2}, S_{2}(R) \eta_{2}\right)$ for $1 \leq j \leq k-1$.

We are in conditions of declare the following system of recurrence relations:

$$
\begin{aligned}
\beta_{n} & =\beta_{1}, \\
\eta_{n} & =\frac{1}{2} h_{n-1}^{k} R^{k-1} \eta_{n-1} \\
h_{n} & =\beta_{n} K \eta_{n},
\end{aligned}
$$




$$
S_{n}(R)=\left\{\begin{array}{l}
1 \quad \text { if } \mathrm{k}=1 \\
1+\frac{h_{n}}{2}\left(1+h_{n} R+\ldots+\left(h_{n} R\right)^{k-2}\right) \text { if } \mathrm{k}>1
\end{array}\right.
$$

The previous study drives us to establish the following result:

Lemma 4 If we assume the hypotheses of lemma 2, then $x_{n}^{(j)}, x_{n+1} \in$ $B\left(x_{n}, S_{n}(R) \eta_{n}\right), 1 \leq j \leq k-1$.

\section{Proof:}

By a similar process that for cases $n=1,2$, we have that for $n \in N$ :

$$
\begin{aligned}
& \left\|F\left(x_{n}^{(1)}\right)\right\| \leq \frac{1}{2} K \eta_{n}\left\|x_{n}^{(1)}-x_{n}^{(0)}\right\| \\
& \left\|F\left(x_{n}^{(j)}\right)\right\| \leq K R \eta_{n}\left\|x_{n}^{(j)}-x_{n}^{(j-1)}\right\|, \quad 2 \leq j \leq k,
\end{aligned}
$$

and for $1 \leq j \leq k-1$ :

$$
\begin{aligned}
\left\|x_{n}^{(j+1)}-x_{n}^{(j)}\right\| & \leq h_{n} R\left\|x_{n}^{(j)}-x_{n}^{(j-1)}\right\| \leq \frac{1}{2} h_{n}^{j} R^{j-1}\left\|x_{n}^{(j)}-x_{n}^{j-1}\right\|, \\
\left\|x_{n}^{(j+1)}-x_{n}^{(0)}\right\| & \leq\left(1+\frac{1}{2} h_{n}+\frac{1}{2} h_{n}^{2} R+\ldots+\frac{1}{2} h_{n}^{j} R^{j-1}\right) \eta_{n} .
\end{aligned}
$$

Then, it follows:

$$
\left\|F\left(x_{n}^{(k-1)}\right)\right\| \leq K R \eta_{n}\left\|x_{n}^{(k-1)}-x_{n}^{(k-2)}\right\|,
$$

and

$$
\begin{aligned}
& \left\|x_{n}^{(k)}-x_{n}^{(k-1)}\right\|=\left\|x_{n+1}-x_{n}^{(k-1)}\right\| \leq h_{n} R\left\|x_{n}^{(k-1)}-x_{n}^{(k-2)}\right\| \leq \frac{1}{2} h_{n}^{k-1} R^{k-2}\left\|x_{n}^{(1)}-x_{n}^{(0)}\right\|, \\
& \left\|x_{n}^{(k)}-x_{n}^{(0)}\right\|=\left\|x_{n+1}-x_{n}^{(0)}\right\| \leq\left(1+\frac{1}{2} h_{n} \frac{1}{2} h_{n}^{2} R+\ldots+\frac{1}{2} h_{n}^{k-1} R^{k-2}\right) \eta_{n} .
\end{aligned}
$$

So, the result is obtained.

In the following lemma we obtain some basic property for the scalar sequences defined in the recurrence relations.

Lemma 5 If $1<R<\frac{2-\sqrt{2 h_{0}}}{2 h_{0}}$, then $\left\{h_{n}\right\}$ and $\left\{S_{n}(R)\right\}$ are decreasing scalar sequences, $n \in \mathbb{N}$.

\section{Proof:}

By the hypothesis, we deduce that $h_{0} R<1$. Then

$$
h_{1}=\beta_{1} K \eta_{1}=\frac{\beta}{1-h_{0} R} K \frac{1}{2\left(1-h_{0} R\right)} h_{0}^{k} R^{k-1} \eta_{0}=\frac{h_{0}}{2} \frac{h_{0}^{k} R^{k-1}}{\left(1-h_{0} R\right)^{2}}<\frac{h_{0}}{2\left(1-h_{0} R\right)^{2}} h_{0}<h_{0},
$$


since that $\frac{h_{0}}{2\left(1-h_{0} R\right)^{2}}<1$ when $R<\frac{2-\sqrt{2 h_{0}}}{2 h_{0}}$.

On the other hand, by using $\beta_{2}=\beta_{1}$, it follows that:

$$
h_{2}=\beta_{2} K \eta_{2}=\beta_{1} K \frac{1}{2} h_{1}^{k} R^{k-1} \eta_{1}=h_{1} \frac{h_{1}}{2}\left(h_{1} R\right)^{k-1}<h_{1},
$$

where in the last inequality we have used that $h_{1}<h_{0}$. Analogously, by an induction procedure, it follows that the sequence $\left\{h_{n}\right\}$ is a decreasing scalar sequence.

Notice that, from $1<R<\frac{2-\sqrt{2 h_{0}}}{2 h_{0}}$ we obtain $\frac{h_{0}}{2}<1$, this fact establish certain relation between our restriction for $R$ and condition $\left(C_{3}\right)$, (pag 5), assumed by Ortega.

Now, by using the previous result, obviously the sequence $S_{n}(R)$ also is a decreasing scalar sequence:

$S_{n}(R)=1+\frac{h_{n}}{2}\left(1+h_{n} R+\ldots+\left(h_{n} R\right)^{k-2}\right)<1+\frac{h_{n-1}}{2}\left(1+h_{n-1} R+\ldots+\left(h_{n-1} R\right)^{k-2}\right)=S_{n-1}(R)$,

for all $n \in N$.

\subsection{Main Result}

Now, for completing the semilocal convergence study we have to prove the assumed assertions that we have made in our previous dissertation, (see Lemma 1 ), which are, $x_{n}^{(j)} \in B\left(x_{0}, R \eta_{n}\right)$, for $1 \leq j \leq k$ and for all $n \in N$, by defining the parameter $R$. For this, we observe that if $1<R<\frac{2-\sqrt{2 h_{0}}}{2 h_{0}}$, then $h_{0} R<1$. Therefore, for $1 \leq j \leq k$ and for all $n \in N$, we have:

$$
\begin{aligned}
\left\|x_{n}^{(j)}-x_{0}\right\| & \leq\left\|x_{n}^{(j)}-x_{n}^{(0)}\right\|+\sum_{j=0}^{n-1}\left\|x_{n-j}^{(0)}-x_{n-j-1}^{(0)}\right\| \leq \sum_{j=0}^{n} S_{j}(R) \eta_{j} \leq S_{0}(R) \sum_{j \geq 0} \eta_{j} \\
& =S_{0}(R)\left[\eta+\frac{\left(h_{0} R\right)^{k-1}}{2\left(1-h_{0} R\right)} h_{0} \eta+\frac{1}{2} h_{1}^{k} R^{k-1} \eta_{1}+\frac{1}{2} h_{2}^{k} R^{k-1} \eta_{2}+\ldots\right] \\
& =S_{0}(R)\left[\eta+\frac{\left(h_{0} R\right)^{k-1}}{2\left(1-h_{0} R\right)} h_{0} \eta+\frac{1}{2} h_{1}^{k} R^{k-1} \eta_{1}+\frac{1}{2} h_{2}^{k} R^{k-1} \frac{1}{2} h_{1}^{k} R^{k-1} \eta_{1}+\ldots\right] \\
& =S_{0}(R)\left[\eta+\frac{\left(h_{0} R\right)^{k-1}}{2\left(1-h_{0} R\right)} h_{0} \eta\left(1+\frac{1}{2} h_{0}^{k} R^{k-1}+\left(\frac{1}{2} h_{0}^{k} R^{k-1}\right)^{2}+\ldots+\left(\frac{1}{2} h_{0}^{k} R^{k-1}\right)^{n}+\ldots\right)\right. \\
& \leq S_{0}(R)\left[1+\frac{\left(h_{0} R\right)^{k-1}}{2\left(1-h_{0} R\right)} h_{0} \frac{1}{1-\frac{1}{2} h_{0}^{k} R^{k-1}}\right] \eta \\
& \leq S_{0}(R)\left[1+\frac{h_{0}^{k} R^{k-1}}{\left(1-h_{0} R\right)\left(2-h_{0}^{k} R^{k-1}\right)}\right] \eta
\end{aligned}
$$


where we have used that $\left\{h_{n}\right\}$ is a decreasing scalar sequence and added the sum of a geometric sequence of reason $\frac{1}{2} h_{0}^{k} R^{k-1}$, which is less than 1 by the hypothesis. So, the previous calculations let us to define an equation to obtain the parameter $R$, defined in theorem 1 , that we write again in order to prove it.

Theorem 6 Let $F$ is a nonlinear operator, $F: \Omega \subseteq X \longrightarrow Y$, defined on a nonempty open convex domain $\Omega$ of a Banach space $X$ with values in a Banach space $Y$. Suppose that conditions $\left(C_{1}\right)-\left(C_{2}\right)$ are satisfied. For a fixed number of steps $k$, if the equation

$$
t=S_{0}(t)\left[1+\frac{h_{0}^{k} t^{k-1}}{\left(1-h_{0} t\right)\left(2-h_{0}^{k} t^{k-1}\right)}\right],
$$

has at least one positive real root and the smallest positive real root, denoted by $R$, satisfies

$$
\frac{h_{0}}{2\left(1-h_{0} R\right)^{2}}<1
$$

and $B\left(x_{0}, R \eta\right) \subset \Omega$, then iterative process given by (3), converges to a solution $x^{*}$ of the equation $F(x)=0$, starting at $x_{0}$, and $x_{n}^{(j)}, x^{*} \in \overline{B\left(x_{0}, R \eta\right)}$, for $1 \leq j \leq k$ and for all $n \in \mathbb{N}$. Moreover the solution $x^{*}$ is unique in $B\left(x_{0}, \frac{2}{K \beta}-R \eta\right) \cap \Omega$.

\section{Proof:}

In first place, from lemma 2 and the previous results, it is obvious that, for all $n \in N$ and $1 \leq j \leq k$, we obtain that $x_{n}^{(j)}$ and $x_{n}^{(k)}=x_{n}$ belong to $B\left(x_{0}, R \eta\right)$.

Once we have seen that the sequence given by (3) is well-defined, we see that it is a Cauchy sequence. Indeed, since

$$
\begin{aligned}
\left\|x_{n+m}-x_{n}\right\| & \leq \sum_{j=1}^{m}\left\|x_{n+j}-x_{n+j-1}\right\| \leq \sum_{j=1}^{m} S_{n+j-1}(R) \eta_{n+j-1} \leq S_{0}(R) \sum_{j=1}^{m} \eta_{n+j-1} \\
& =S_{0}(R) \sum_{p=0}^{m-1} \eta_{n+p} \leq S_{0}(R) \sum_{p=0}^{m-1}\left(\frac{h_{0}}{2}\right)^{n+p}\left(h_{0} R\right)^{(n+p)(k-1)} \eta_{0}<S_{0}(R) \sum_{p=0}^{m-1}\left(\frac{h_{0}}{2}\right)^{n+p} \eta_{0} \\
& \leq S_{0}(R) \frac{\left(\frac{h_{0}}{2}\right)^{n}-\left(\frac{h_{0}}{2}\right)^{n+m}}{1-\frac{h_{0}}{2}} \eta_{0},
\end{aligned}
$$

Consequently, the sequence $\left\{x_{n}\right\}$ is convergent. Now, if $\lim _{n \rightarrow \infty} x_{n}=x^{*}$, it 
follows that $F\left(x^{*}\right)=0$ from the continuity of the operator $F$, since

$$
\begin{aligned}
\left\|F\left(x_{n}\right)=\right\| F\left(x_{n-1}^{(k)}\right) \| & \leq K R \eta_{n-1}\left\|x_{n-1}^{(k)}-x_{n-1}^{(k-1)}\right\| \leq K R \eta_{n-1} \frac{1}{2} h_{n-1}^{k-1} R^{k-2}\left\|x_{n-1}^{(1)}-x_{n-1}^{(0)}\right\| \\
& \leq \frac{1}{2} K\left(h_{n-1} R\right)^{k-1} \eta_{n-1}^{2} \leq \frac{1}{2} K \eta_{n-1}^{2} \leq \frac{1}{2} K\left(\left(\frac{h_{0}}{2}\right)^{n-1} \eta_{0}\right)^{2}
\end{aligned}
$$

and $\left(\frac{h_{0}}{2}\right)^{n-1} \rightarrow 0$ by letting $n \rightarrow \infty$.

To prove the uniqueness, let us assume some other solution $z^{*}$ of $F(x)=0$ in $B\left(x_{0}, \frac{2}{K \beta}-R \eta\right) \cap \Omega$. From the approximation

$$
F\left(z^{*}\right)-F\left(x^{*}\right)=\int_{0}^{1} F^{\prime}\left(x^{*}+t\left(z^{*}-x^{*}\right)\right) d t\left(z^{*}-x^{*}\right)=0
$$

we have to prove that the operator $\int_{0}^{1} F^{\prime}\left(x^{*}+t\left(z^{*}-x^{*}\right)\right) d t$ is invertible and then $z^{*}=x^{*}$. Indeed, from

$$
\begin{aligned}
& \left\|\Gamma_{0} \int_{0}^{1} F^{\prime}\left(x^{*}+t\left(z^{*}-x^{*}\right)\right) d t-I d\right\| \leq\left\|\Gamma_{0}\right\| \int_{0}^{1}\left\|F^{\prime}\left(x^{*}+t\left(z^{*}-x^{*}\right)\right)-F^{\prime}\left(x_{0}\right)\right\| d t \\
& \leq K \beta \int_{0}^{1}\left\|x^{*}+t\left(z^{*}-x^{*}\right)-x_{0}\right\| d t \leq K \beta \int_{0}^{1}\left((1-t)\left\|x^{*}-x_{0}\right\|+t\left\|z^{*}-x_{0}\right\|\right) d t<1,
\end{aligned}
$$

it follows that $\left[\int_{0}^{1} F^{\prime}\left(x^{*}+t\left(z^{*}-x^{*}\right)\right) d t\right]^{-1}$ exists.

Notice that, as $h_{0} R<1$, then $\frac{2}{K \beta}-R \eta>0$.

\section{On the accessibility of the iterative process}

In this section we consider conditions imposed in Theorem 1, given by (12)(18) for the starting point $x_{0}$, in order to analyze when the semilocal convergence of iterative process (3) is guaranteed. That is, we obtain the domain of starting points for our iterative process. For this, we consider the smallest positive real root of the equation (12), denoted by $R$, and the parameter $h_{0}$ associated with the starting point $x_{0}$. Then, in first place, we study when the equation (12) has solution. Second, we will analyze the relationship between the parameters $R$ and $h_{0}$ from condition (18). Note that the existence of $R$ depends on the value $h_{0}$, that we get from $x_{0}$, and the number of prefixed steps, $k$.

The aim of Figure 1 is to see when the equation (12) has solution from the variability of $k$ and $h_{0}$. So, in this case, we consider the steps $k=6,8,10,15$ 

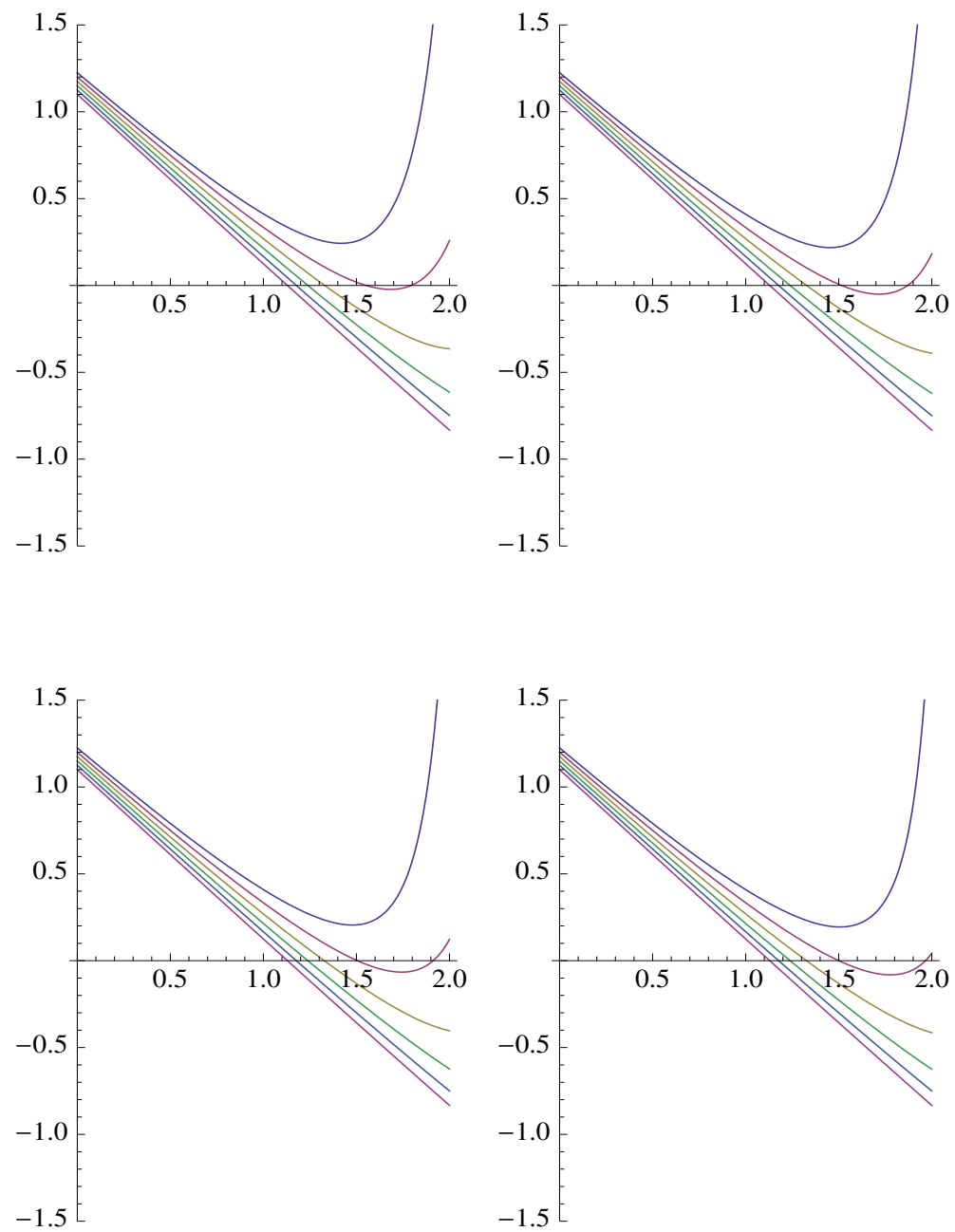

Figure 1: Equation (12) for $k=6,8,10,15$ and $h_{0}=$ $0.45,0.4,0.35,0.3,0.25,0.2$.

for the values $h_{0}=0.45$ (purple line), $h_{0}=0.40$ (brown line), $h_{0}=0.35$ (yellow line), $h_{0}=0.30$ (green line), $h_{0}=0.25$ (blue line) and $h_{0}=, 0.20$ (pink line). We can observe that in the case of $h_{0}=0.45$ there is not root of the equation (12) and so we can not apply Theorem 1 . However, below the value $h_{0}=0.4$ we can see that there is always $R$.

Now, we see the relationship between the parameters $h_{0}$ and $R$ associated with the the starting point $x_{0}$ and the smallest positive real root of the equation (12) respectively. By choosing $x=h_{0}$ and $y=R$, we can draw the region of the $x y$-plane whose points satisfy the condition (18) (namely, 


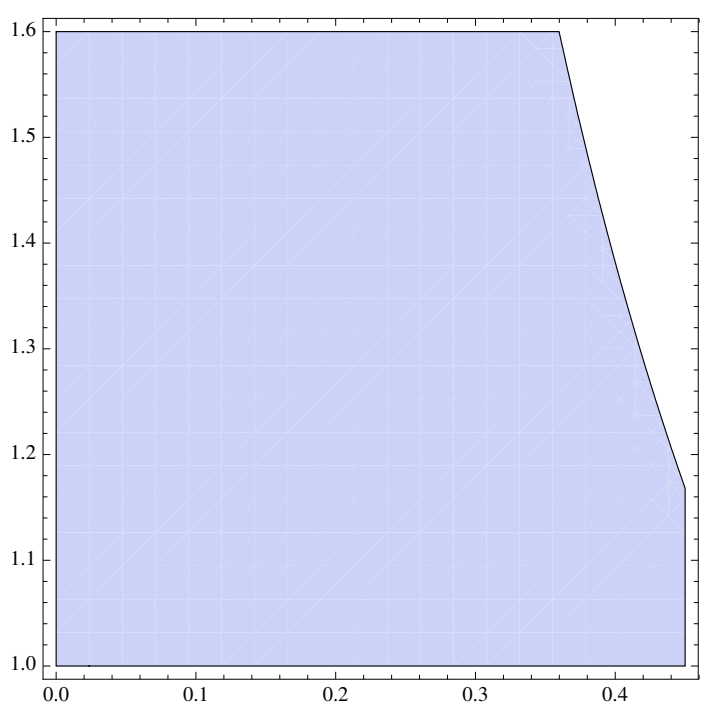

Figure 2: The relationship between the parameters $h_{0}$ and $R$ given by (18).

$\left.\frac{x}{2(1-x y)^{2}}<1\right)$. This condition indicates the domain of parameters for the method (3). Moreover, notice that the convergence of the iterative process is guaranteed from this condition imposed in Theorem 1 that is shown in Figure 2 (cyan region).
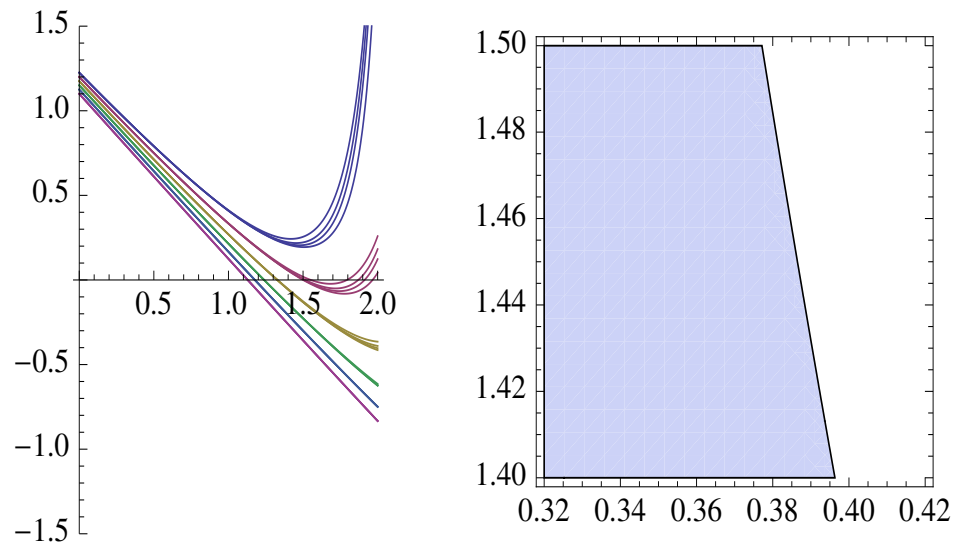

Figure 3: Graphics for condition (12) for $k=6,8,10,15$ and domain of parameters of the condition (18) for values close to $h_{0}=0.4$.

In relation to the above, we can think that the larger the size of the domain of parameters is, we have more possibilities for choosing good starting points for iterative process (3), provided we have tested the existence of $R$. 
As we have already seen in Figure 1, to values lower than 0.4 always exists the $R$ value. However, note that when $h_{0}=0.4$, the value of $R$ is approximately 1.5, then, if we look at Figure 2, we see that for these parameter values cannot be verified the condition (18). This fact can be seen more clearly at Figure 3, where we have drawn all the graphs obtained from the equation (12) and given in Figure 1, seeing that for values lower than $h_{0}=0.4$ always it will exist $R$. However, for $h_{0}$ values close to this value may be not checked the condition (18).

In addition, noting the domain of parameters given in the Figure 4 , to values lower than $h_{0}=0.4$ there exists $R$ and also verifies the condition (18), as you can clearly see in this figure.

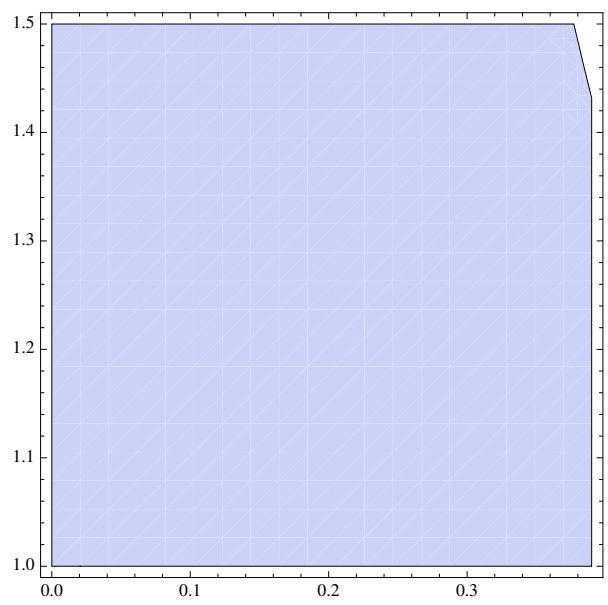

Figure 4: Domain of parameters of the condition (18) for values close to $h_{0}=0.38$.

\section{On the numerical resolution of a special kind of conservative problems}

We center our attention on a conservative system like gravitational and electric fields. System in which work done by a force is independent of the path taken and is equal to the difference between the final and initial values of an energy function.

Specifically, we consider a conservative system where the damping force is null and then can be described as follows:

$$
\frac{d^{2} x(t)}{d t^{2}}+\phi(x(t))=0,
$$


where $x(t) \in C^{(2}[a, b]$, with boundary conditions

$$
x(a)=A, x(b)=B .
$$

Boundary value problems have plenty of applications in science and engineering, as can be found in classical references [2] and [12].

Now, we first do a theoretical study of the formulated problem. So, from the continuous case, we study the existence and uniqueness of solution for problem (14)-(15) from the previous analysis. From optimizing the parameter $k$, we obtain the optimal domains of existence and uniqueness of solution. After that, problem (14)-(15) is discretized and a solution of the problem is numerically approximated. For this, from using the location of the solution previously done in the continuous case, we choose the optimal number of steps of iterative process (3) that provides the most efficient iterative process for approximating numerically a solution of problem (14)-(15) once it is discretized.

\subsection{Existence and uniqueness of the solution}

It is well known that the solution of (14)-(15) is a solution of the following Fredholm integral equation (see [16]).

$$
x(s)=\int_{a}^{b} G(s, t) \phi(x(t)) d t+\frac{B-A}{b-a} s+\frac{b A-a B}{b-a},
$$

where the kernel $G$ is the Green function in $[a, b] \times[a, b]$ :

$$
G(t, s)= \begin{cases}\frac{(b-s)(t-a)}{b-a}, & t \geq s \\ \frac{(s-a)(b-t)}{b-a}, & t \leq s .\end{cases}
$$

A technique to solve these kind of equation consist in express it in a Banach space as a nonlinear operator, that is: $F(x)=0$, where $F: \Omega \subseteq$ $C[a, b] \rightarrow C[a, b]$ and

$$
[F(x)](s)=x(s)-\int_{a}^{b} G(s, t) \phi(x(t)) d t-\frac{B-A}{b-a} s-\frac{b A-a B}{b-a},
$$

considering the max-norm $\|\nu\|=\max _{s \in[a, b]}|\nu(s)|$ in $C[a, b]$.

To obtain the existence of a unique solution of (17) by theorem 1, observe that we need to evaluate $K, \beta$ and $\eta$ from the initial point $x_{0}$, and define the domain $\Omega$. 
In first place, we calculate the first Frechet derivative of the operator given by (17) :

$$
\left[F^{\prime}(x)\right] y(s)=y(s)-\int_{a}^{b} G(s, t) \phi^{\prime}(x(t)) y(t) d t
$$

then, we have:

$$
\left\|I-F^{\prime}\left(x_{0}\right)\right\| \leq \frac{M}{8},
$$

where $\max _{t \in[0,1]} \int_{0}^{1}|G(s, t)| d t=\frac{1}{8}$ and $M=\max _{t \in[0,1]}\left|\phi^{\prime}\left(x_{0}(t)\right)\right|$.

So, by Banach lemma, if $M<8$ then $\Gamma_{0}=F^{\prime}\left(x_{0}(t)\right)^{-1}$ exists and $\left\|\Gamma_{0}\right\|<$ $\frac{8}{8-M}$. In this situation, we consider $\Omega=\left\{x \in C[a, b] / \max _{t \in[0,1]}\left|\phi^{\prime}(x(t))\right|<8\right\}$. Moreover, we obtain:

$$
\left\|\Gamma_{0} F\left(x_{0}\right)\right\| \leq \frac{8\left\|F\left(x_{0}\right)\right\|}{8-M}
$$

In second place, if $\tilde{K}$ is the Lipschitz constant for $\phi^{\prime}$, it follows

$$
\left\|F^{\prime}(x)-F^{\prime}(y)\right\| \leq \frac{\tilde{K}}{8}\|x-y\| .
$$

Finally, for an initial point $x_{0}(t)$, we obtain:

$$
a=\beta K \eta=\frac{8 \tilde{K}\left\|F\left(x_{0}\right)\right\|}{(8-M)^{2}} .
$$

Consequently, a more explicit result on the existence and uniqueness of a solution of (17) is given in the next theorem, whose proof follows from that of theorem 1 .

Theorem 7 Following the previous notations, considering that $x_{0} \in \Omega$ and $\tilde{K}$ is the Lipschitz constant for $\phi^{\prime}$, assume that for a fixed number of steps $k$, the polynomial equation: $P(k, t)=0$, where

$$
P(k, t)=\left\{\begin{array}{l}
2-\left(2+a-a^{2}\right) t+a(2-a) t^{2} \text { if } \mathrm{k}=1 \\
2+a-2(1+a) t-2 a t^{2}-a^{k} t^{k-1}+(1+a) a^{k} t^{k}-a^{k+1} t^{k+1} \\
+\frac{1}{2} a^{k+2} t^{k} \sum_{j=0}^{k-2} a^{j} t^{j} \text { if } \mathrm{k}>1
\end{array}\right.
$$

has at least one positive real root and the smallest positive real root, denoted by $R$, satisfies

$$
\frac{a}{2(1-a R)^{2}}<1
$$

If $B\left(x_{0}, R \eta\right) \subseteq \Omega$, then iterative process given by (3), converges to a solution $x^{*}$ of the equation $F(x)=0$, starting at $x_{0}$, and $x_{n}^{(j)}, x^{*} \in \overline{B\left(x_{0}, R \eta\right)}$, for $1 \leq j \leq k$ and for all $n \in \mathbb{N}$. Moreover the solution $x^{*}$ is unique in $B\left(x_{0}, \frac{2}{K \beta}-\right.$ $R \eta) \cap \Omega$. 


\section{A particular case}

Nonlinear differential equations of second-order occur frequently in connection with applied problems, a circumstance which has led to considerable interest in them in recent years. We show here the application to a simple example that has been studied by Jerome and Varga [9] and Keller [11]. The problem is:

$$
\begin{aligned}
& \frac{d^{2} x(t)}{d t^{2}}=e^{x(t)} \\
& x(0)=0=x(1) .
\end{aligned}
$$

Therefore, following the previous study, we consider the operator

$$
[F(x)](s)=x(s)-\int_{0}^{1} G(s, t) e^{x(t)} d t
$$

in the space $C[0,1]$ of all continuous functions with the max-norm. To obtain a result on the existence and uniqueness of solutions of (20), we first consider

$$
\Omega=\{x \in C[0,1]:\|x\|<\ln 8\} \subseteq C[0,1]
$$

and take $F: \Omega \subseteq C[0,1] \rightarrow C[0,1]$.

According to standard differential equation (14), we have $\phi(x(t))=-e^{x(t)}$. Then, for $x_{0} \in \Omega$ and by the previous study, we have that $\Gamma_{0}$ exists and

$$
\left\|\Gamma_{0}\right\| \leq \frac{8}{8-M}
$$

where $M=\max _{t \in[0,1]} e^{x_{0}(t)}$, and

$$
\left\|\Gamma_{0} F\left(x_{0}\right)\right\| \leq \frac{8\left\|F\left(x_{0}\right)\right\|}{8-M}
$$

On the other hand, it is easy to check

$$
\left\|F^{\prime}(x)-F^{\prime}(y)\right\| \leq\|x-y\|, \quad x, y \in \Omega,
$$

therefore $K=\frac{\tilde{K}}{8}=1$.

From (19), the solution is a convex function and satisfies the boundary conditions. We then consider the simplest case, a 2nd degree polynomial of the form $m t(t-1), m \geq 0$, as a first approximation to the solution of (19). How it should be verified that $\|m t(t-1)\|<\ln 8$, for simplicity, we choose $m=1 / 2$, that is, $x_{0}(t)=\frac{1}{2} t(t-1)$. Thus 


$$
M=1 \quad \text { and } \quad\left\|F\left(x_{0}\right)\right\| \leq\left\|\frac{1}{2} t(1-t)\right\|+\frac{1}{8} \int_{0}^{1} d t=1 / 4 .
$$

Therefore $a=16 / 49$ and then in Table 1 we can see the corresponding radii of existence and uniqueness for different values of $k$, as can be observed the best results are obtained for $k=2$, that correspond to the third order method. In this case we obtain the smallest ball where the iterates remains and converge to the solution in this case the biggest ball for the uniqueness is also obtained. Notice that all the results improve the ones obtained for Newton's method.

\begin{tabular}{|c|c|c|}
\hline Steps number & Convergence radius & Uniqueness radius \\
\hline$k$ & $R \eta$ & $\frac{2}{K \beta}-R \eta$ \\
\hline 1 & 0.38533426 & 1.36466574 \\
$\mathbf{2}$ & $\mathbf{0 . 2 1 0 8 8 3 8 7}$ & $\mathbf{1 . 5 3 9 1 1 6 1 3}$ \\
3 & 0.21165205 & 1.53834795 \\
4 & 0.21182150 & 1.5381785 \\
5 & 0.21186161 & 1.53813839 \\
6 & 0.21187127 & 1.53812873 \\
7 & 0.21187360 & 1.5381264 \\
\hline
\end{tabular}

Table 1: Convergence and uniqueness radii for different $\mathrm{k}$-steps iterative methods.

That is, we have obtained that the iterates $x_{n}(t)$ and the solution $x^{*}(t)$ belong to $\overline{B\left(\frac{1}{2} t(t-1), 0.21088387\right)} \subseteq \Omega$, and $x^{*}(t)$ is the unique solution in $B\left(\frac{1}{2} t(t-1), 1.53911613\right) \cap \Omega$. Then, we have the solution localized and so we have also an idea in order to take starter conditions.

\subsection{Setting up a finite difference scheme}

After the study we have just performed for the continuous case, we use a discretization process to transform equations (14)-(15) into a finite-dimensional problem and look for an approximated solution of this when a particular function $\phi(x(t))$ is considered. For this purpose we approximate the second derivative by a standard numerical formula.

First, we introduce the points $t_{j}=a+j(b-a) h, j=0,1, \ldots, N+1$, where $h=\frac{1}{N+1}$ and $N$ is an appropriate integer. A scheme is then designed for the determination of numbers $x_{j}$, it is hoped, approximate the values $x\left(t_{j}\right)$ of the true solution at the points $t_{j}$. A standard approximation for the 
second derivative at these points is

$$
x_{j}^{\prime \prime} \approx \frac{x_{j-1}-2 x_{j}+x_{j+1}}{h^{2}}, \quad j=1,2, \ldots, N .
$$

A natural way to obtain such a scheme is to demand that the $x_{j}$ satisfy at each interior mesh point $t_{j}$ the difference equation

$$
x_{j-1}-2 x_{j}+x_{j+1}+h^{2} \phi\left(x_{j}\right)=0 .
$$

Since $x_{0}$ and $x_{N+1}$ are determined by the boundary conditions, the unknowns are $x_{1}, x_{2}, \ldots, x_{N}$.

A further discussion is simplified by the use of matrix and vector notation. Introducing the vectors

$$
\mathbf{x}=\left(x_{1}, x_{2}, \ldots, x_{N}\right)^{t}, \quad v_{\mathbf{x}}=\left(\phi\left(x_{1}\right), \phi\left(x_{2}\right), \ldots, \phi\left(x_{N}\right)\right)^{t}
$$

and the matrix

$$
A=\left(\begin{array}{ccccc}
-2 & 1 & 0 & \cdots & 0 \\
1 & -2 & 1 & \cdots & 0 \\
0 & 1 & -2 & \cdots & 0 \\
\vdots & \vdots & \vdots & \ddots & \vdots \\
0 & 0 & 0 & \cdots & -2
\end{array}\right)
$$

the system of equations, arising from demanding that (22) holds for $j=$ $1,2, \ldots, N$, can be written compactly in the form:

$$
F(\mathbf{x}) \equiv A \mathbf{x}+h^{2} v_{\mathbf{x}}=0,
$$

where $F$ is a function from $\mathbb{R}^{N}$ into $\mathbb{R}^{N}$.

From now on, the focus of our attention is to solve a particular system of form (23). As, in general, we consider that the function $\phi(x(t))$ is nonlinear, one cannot hope to solve the system of equations given in (23) by algebraic methods. Some iterative procedure must be resorted to. We now analyze the family of iterative processes given by (3) for this purpose. First, note that the practical application of these iterative processes is performed from the following algorithm, depending on the chosen number of steps.

$$
\left\{\begin{array}{l}
F^{\prime}\left(x_{n}\right)\left(x_{n}^{(1)}-x_{n}^{(0)}\right)=-F\left(x_{n}^{(0)}\right), \\
F^{\prime}\left(x_{n}\right)\left(x_{n}^{(2)}-x_{n}^{(1)}\right)=-F\left(x_{n}^{(1)}\right), \\
\quad \vdots \\
F^{\prime}\left(x_{n}\right)\left(x_{n}^{(k-1)}-x_{n}^{(k-2)}\right)=-F\left(x_{n}^{(k-2)}\right), \\
F^{\prime}\left(x_{n}\right)\left(x_{n}^{(k)}-x_{n}^{(k-1)}\right)=-F\left(x_{n}^{(k-1)}\right), \quad n \geqslant 0,
\end{array}\right.
$$


Notice that the first derivative of $F$ is now the matrix

$F^{\prime}(\mathbf{x})=A+h^{2} \operatorname{diag}\left(v_{\mathbf{x}}^{\prime}\right), \quad v_{\mathbf{x}}^{\prime}=\left(v_{1}^{\prime}, v_{2}^{\prime}, \ldots, v_{8}^{\prime}\right)^{t}, \quad v_{i}^{\prime}=-e^{x_{i}}, \quad i=1,2, \ldots, N$.

Now we are going to choose the steps number that we will perform with the iterative method (3) so that maximum efficiency is reached.

To do this, we consider a combination of indexes considered previously, in (2), the efficiency index and the computational efficiency index. Note that, if we consider a particular problem, we can calculate the operational cost required to evaluate $F$ and $F^{\prime}$. So, we consider another measure of the efficiency of an iterative process which takes into account both the operational cost of the functional evaluations that are required and the operational cost of doing an step of the algorithm.

Thus, we define the measure of the efficiency of an iterative process applied to an operator $F$ given as follows

$$
E(\operatorname{method}(3), F)=(k+1)^{1 /(\mu+\sigma)},
$$

where the operational cost of the functional evaluations and the operational cost of doing an step of the algorithm are denoted by $\mu$ and $\sigma$, respectively.

Once we have chosen the number of steps making optimum efficiency of the iterative process (3), then we solve the nonlinear system raised previously.

\section{A particular case}

Now our goal is to find an approach to a solution of the differential problem defined in (19). In this case the number of operations related to evaluate $F\left(x_{n}\right)$ are $3 N+1$ and $F^{\prime}\left(x_{n}\right)$ does not need any new operation. As each iteration of the iterative methods (3) require $\left(N^{3}+3 k N^{2}-N\right) / 3$ operations, we obtain:

$$
E(\operatorname{method}(3), F)=(k+1)^{\frac{3}{3 k\left(N^{2}+3 N\right)+N^{3}-N+3}} .
$$

Then by taking different values of $N$, we observe in Figure 5 the variations of the efficiency for different values of $k$. Observe that when $N$ increases the maximum efficiency is reached by performing more steps, we find that for $N=20$ the most efficient method correspond to $k=5$ and for $N=25,30,35$ the maximum efficiency is obtained for $k=6,7,8$, respectively. Notice that $k=1$, Newton's method, reach for all values of $N$ the worst efficiency.

For solving (23) as is expressed in (24) with function $\phi(x)=-e^{x(t)}$ we work with $N=20$ and $k=5$, then by taking as started approximation the discretization of the function $x_{0}(t)=\frac{1}{2} t(t-1)$ in $[0,1]$ we obtain after 3 iterations the results of Tables 2 and 3, by working with variable precision arithmetic of 200 digits. 


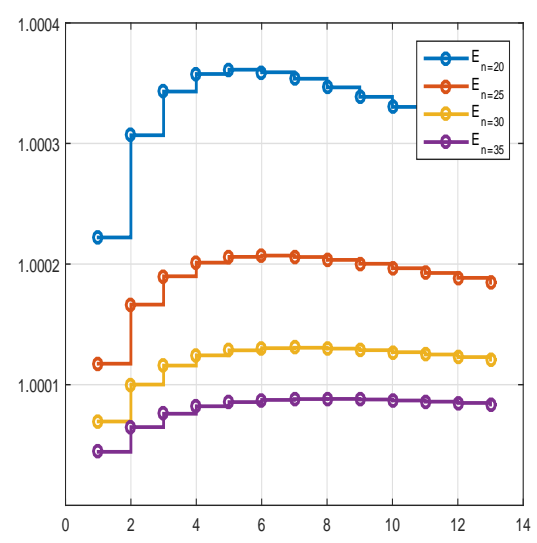

Figure 5: Efficiency for $k=1: 13$ and $20 \leq N \leq 35$.

\begin{tabular}{|c|c|c|c|c|c|}
\hline \hline$i$ & $x_{i}^{*}$ & $i$ & $x_{i}^{*}$ & $i$ & $x_{i}^{*}$ \\
\hline 1 & $-0.0209484 \ldots$ & 9 & $-0.111408 \ldots$ & 17 & $-0.0706296, \ldots$ \\
2 & $-0.0396762 \ldots$ & 10 & $-0.113432 \ldots$ & 18 & $-0.0562247, \ldots$ \\
3 & $-0.0562247 \ldots$ & 11 & $-0.113432 \ldots$ & 19 & $-0.0396762 \ldots$ \\
4 & $-0.0706296 \ldots$ & 12 & $-0.111408 \ldots$ & 20 & $-0.0209484 \ldots$ \\
5 & $-0.0829215 \ldots$ & 13 & $-0.107355 \ldots$ & & \\
6 & $-0.0931263 \ldots$ & 14 & $-0.101265 \ldots$ & & \\
7 & $-0.101265 \ldots$ & 15 & $-0.0931263 \ldots$ & & \\
8 & $-0.107355 \ldots$ & 16 & $-0.0829215 \ldots$ & & \\
\hline \hline
\end{tabular}

Table 2: Numerical solution $\mathbf{x}^{*}$ of $(23)$ with $\phi(x)=-e^{x(t)}$.

\section{Conclusion}

We have obtained semilocal convergence study for k-steps iterative method, this is a generic study that requires a procedure more intrincate that the one needed for a fixed convergence order method. We can conclude that the numerical experience confirm the theoretical study performed in section 2, allowing us to obtain the convergence ball where the approximation to the solution of a nonlinear problem has been obtained by using the most efficient iterative method of the $\mathrm{k}$-steps iterative process with frozen first derivative described by (3). Moreover the accessibility of the iterative process have been analyzed obtaining the domain of starting points for setting the semilocal convergence of our iterative process. 


\begin{tabular}{|c||c|c|}
\hline \hline$n$ & $\left\|\mathbf{x}^{*}-\mathbf{x}_{\mathbf{n}}\right\|$ & $\left\|F\left(\mathbf{x}_{\mathbf{n}}\right)\right\|$ \\
\hline 0 & $4.76 \times 10^{-2}$ & $3.86 \times 10^{-2}$ \\
1 & $1.13 \times 10^{-18}$ & $3.71 \times 10^{-20}$ \\
2 & $6.19 \times 10^{-117}$ & $1.57 \times 10^{-118}$ \\
3 & 0 & $1.77 \times 10^{-209}$ \\
\hline \hline
\end{tabular}

Table 3: Absolute errors and $\left\{\left\|F\left(\mathbf{x}_{\mathbf{n}}\right)\right\|\right\}$.

\section{References}

[1] S. Amat, S. Busquier, C. Bermúdez and S. Plaza, On two families of high order Newton type methods, Appl. Math. Comput., 25 (2012), 2209-2217.

[2] I.K. Argyros, S. Hilout and M.A. Tabatabai Mathematical Modelling with Applications in Biosciences and Engineering. Nova Publishers, New York (2011).

[3] I. K. Argyros and S. George, A Unified Local Convergence for Jarratttype Methods in Banach Space Under Weak Conditions, Thai J. Math., 13 (2015), 165-176.

[4] I. K. Argyros, and S. Hilout, On the local convergence of fast two-step Newton-like methods for solving nonlinear equations, J. Comput. Appl. Math., 245 (2013), 1-9.

[5] I.K. Argyros, J.A. Ezquerro, J.M. Gutiérrez, M.A. Hernández and S. Hilout On the semilocal convergence of efficient ChebyshevSecant-type methods, J. Comput. Appl. Math., 235 (2011), 3195-2206.

[6] A. Cordero, J. L. Hueso, Eulalia Martínez and J. R. Torregrosa, Generating optimal derivative free iterative methods for nonlinear equations by using polynomial interpolation. Math. Comput. Mod., 57 (2013), 1950-1956.

[7] J. A. Ezquerro, M. Grau-Sánchez, M. A. Hernández and M. Noguera, Semilocal convergence of secant-like methods for differentiable and nondifferentiable operators equations, J. Math. Anal. Appl., 398, 1, (2013), 100-112.

[8] G. Honorato, S. Plaza and N. Romero, Dynamics of a higher-order family of iterative methods, J. Complexity 27, 2 (2011), 221-229. 
[9] J. W. Jerome and R. S. Varga, Generalizations of Spline Functions and Applications to Nonlinear Boundary Value and Eigenvalue Problems, Theory and Applications of Spline Functions. Academic Press, New York (1969).

[10] L. V. Kantorovich and G. P. Akilov, Functional analysis Pergamon Press, Oxford (1982).

[11] H. B. Keller, Numerical Methods for Two-Point Boundary-Value Problems. Dover Publications, New York (1992).

[12] T.Y. Na, Computational Methods in Engineering Boundary Value Problems, Academic Press, New York (1979).

[13] J. M. Ortega, The Newton-Kantorovich theorem, Amer. Math. Monthly, 75 (1968), 658-660.

[14] A. M. Ostrowski, Solutions of Equations in Euclidean and Banach Spaces. Academic Press, New York (1973).

[15] S. Plaza and N. Romero, Attracting cycles for the relaxed Newton's method, J. Comput. Appl. Math., 235, 10 (2011), 3238-3244.

[16] D. Porter and D. Stirling, Integral Equations: A Practical Treatment, From Spectral Theory to Applications. Cambridge University Press, Cambridge (1990).

[17] J. F. Traub, Iterative Methods for the Solution of Equations. PrenticeHall, Englewood Cliffs, New Jersey (1964).

[18] I.K. Argyros, S. George, Extending the applicability of Gauss-Newton method for convex composite optimization on Riemannian manifolds using restricted convergence domains, Journal of Nonlinear Functional Analysis 2016 (2016), Article ID 27.

[19] J.Z. Xiao, J. Sun, X. Huang, Approximating common fixed points of asymptotically quasi-nonexpansive mappings by a $\mathrm{k}+1$-step iterative scheme with error terms, J. Comput. Appl. Math. 233 (2010), 20622070 .

[20] X. Qin, B.A.B. Dehaish, S.Y. Cho, Viscosity splitting methods for variational inclusion and fixed point problems in Hilbert spaces, J. Nonlinear Sci. Appl. 9 (2016), 2789-2797, 